\title{
TILTING MODULES AND THE SUBCATEGORIES $\mathrm{C}_{\mathbf{i}}^{\mathrm{M}}$
}

\author{
María Inés Platzeck, ${ }^{1}{ }^{*}$ and Nilda Isabel Pratti ${ }^{2} \dagger$ \\ ${ }^{1}$ Instituto de Matemática, Universidad Nacional del Sur, \\ 8000 Bahía Blanca, Argentina \\ ${ }^{2}$ Dpto. de Matemática, F.C.E. y N., Universidad Nacional de Mar del Plata, \\ 7600 Mar del Plata, Argentina
}

\begin{abstract}
In this paper we further study the full subcategories $C_{i}^{M}$ of the category of finitely generated modules over an artin algebra introduced in [PP], consisting of the modules having an add $M$ resolution of length $i$, which remains exact under the functor $\operatorname{Hom}_{A}(M,-)$. In particular, we characterize tilting modules in terms of these categories and determine when the transpose of a tilting module is a tilting module.
\end{abstract}

\section{Introduction}

Let $A$ be an artin algebra and $\bmod A$ be the category of finitely generated right $A$-modules. Let $M$ be an $A$-module and denote by add $M$ the full subcategory of $\bmod A$ consisting of the direct sums of direct summands of $M$. In [PP] we considered for an $A$-module $M$ and for every $n \geq 0$ the full subcategories $C_{n}^{M}$ of $\bmod A$ consisting of the modules $X$ such that there is an exact sequence $M_{n} \rightarrow$ $\cdots \rightarrow M_{1} \rightarrow M_{0} \rightarrow X \rightarrow 0$ with $M_{i} \in$ add $M$, and such that the induced sequence $\operatorname{Hom}_{A}\left(M, M_{n}\right) \rightarrow \cdots \rightarrow \operatorname{Hom}_{A}\left(M, M_{1}\right) \rightarrow \operatorname{Hom}_{A}\left(M, M_{0}\right) \rightarrow \operatorname{Hom}_{A}(M, X) \rightarrow 0$ is exact, generalizing work of $\mathrm{M}$. Auslander in [A] about the subcategory $C_{1}^{M}$. The results in [PP] refer mainly to $C_{0}^{M}$ and $C_{1}^{M}$, and the modules $M$ with the property that $C_{0}^{M}=C_{1}^{M}$ are studied there. Examples of such modules are semisimple modules, tilting modules, ${ }^{*}$-modules (as defined en $[\mathrm{C}]$ ) and the transpose of tilting modules.

In this paper we give some applications of the results in $[\mathrm{PP}]$. On one side, we prove that the transpose $\operatorname{Tr} M$ of a tilting module $M$ is a ${ }^{*}$-module. Using then a result by D'Este and Happel about *-modules it follows that $\operatorname{Tr} M$ is a tilting module over the algebra $\operatorname{End}\left({ }_{B} M\right) / P\left({ }_{B} M,_{B} M\right)$, where $B=\operatorname{End}\left(M_{A}\right)$ and $P\left({ }_{B} M,_{B} M\right)$ is the set of the endomorphisms of ${ }_{B} M$ which factor through a projective module. As a consequence we obtain conditions for the transpose of a tilting module $M$ to be a tilting module. This is the case, for example, when $M$ is a splitting or a separating tilting module with no nonzero projective summands.

Tilting modules $M$ satisfy $C_{0}^{M}=C_{1}^{M}$. The converse is not true, even if we assume that $D A \in C_{0}^{M}$. An example is provided by the module $M$ direct sum of a complete set of representatives of the isomorphism classes of indecomposable modules over an algebra of finite representation type. However, we characterize tilting modules in terms of the categories $C_{i}^{M}$ in the following way. Let $B=$

*E-mail platzeck@uns.edu.ar

†E-mail nilprat@mdp.edu.ar 
$\operatorname{End}\left(M_{A}\right)$. The module $M$ is a tilting module if and only if $D A \in C_{0}^{M_{A}}, C_{0}^{M_{A}}=$ $C_{1}^{M_{A}}$ and $C_{0}^{B}=C_{1}^{B}$. We study the relation between the validity of some properties defining a tilting module, and the conditions $C_{0}^{M_{A}}=C_{1}^{M_{A}}$ and $C_{0}^{B}{ }^{M}=$ $C_{1}^{B} M$.

Finally, we consider generalized tilting modules and prove that generalized tilting modules $M$ of projective dimension $n$ have the property that $D A \in C_{n-1}^{M_{A}}$, and the equalities $C_{n-1}^{M_{A}}=C_{n}^{M_{A}}$ and $C_{n-1}^{B}{ }^{B}=C_{n}^{B}{ }^{M}$ hold. However, the converse does not hold.

\section{Preliminaries}

Throughout this paper $A$ denotes an artin algebra, $\bmod A$ the category of finitely generated right $A$-modules and $A^{o p}$ the opposite algebra of $A$. The word module means finitely generated module and we will write $M_{A}$ or $M$ to indicate that the $A$-module $M$ is a right module, and ${ }_{A} M$ to indicate that it is a left module. All subcategories considered are full. We will denote by $D: \bmod A \rightarrow \bmod A^{\text {op }}$ the usual duality for artin algebras. Moreover, $\operatorname{pd} M$ denotes the projective dimension and $\operatorname{id} M$ the injective dimension of the module $M$. We denote by $\operatorname{Tr} M$ the transpose of $M$ and by $\operatorname{Gen} M$ (respectively, $\operatorname{Cogen} M$ ) the subcategory of $\bmod A$ generated (respectively, cogenerated) by $M$.

According to $[\mathrm{HR}, 3]$ we will say that the module $M_{A}$ is a tilting module if it satisfies the following conditions:

(T1) $\operatorname{pd} M_{A} \leq 1$.

(T2) $\operatorname{Ext}_{A}^{1}\left(M_{A}, M_{A}\right)=0$.

(T3) There exists an exact sequence $0 \rightarrow A \rightarrow M^{\prime} \rightarrow M^{\prime \prime} \rightarrow 0$ with $M^{\prime}, M^{\prime \prime}$ in $\operatorname{add} M_{A}$.

It was shown in [BB] and [HR] that if $M$ is a tilting module and $B=\operatorname{End}(M)$ then: 1) ${ }_{B} M$ is a tilting module and $A \simeq \operatorname{End}\left({ }_{B} M\right)$ and 2) the functors $\operatorname{Hom}_{A}(M,-)$ and $-\otimes_{B} M$ induce mutually inverse equivalences between the full subcategories $\mathcal{T}(M)=\left\{X: \operatorname{Ext}_{A}^{1}(M, X)=0\right\}$ and $\mathcal{Y}(M)=\left\{Y: \operatorname{Tor}_{1}^{B}(Y, M)=0\right\}$ while the functors $\operatorname{Ext}_{A}^{1}(M,-)$ and $\operatorname{Tor}_{1}^{B}(-, M)$ induce mutually inverse equivalences between the full subcategories $\mathcal{F}(M)=\left\{X: \operatorname{Hom}_{A}(M, X)=0\right\}$ and $\mathcal{X}(M)=\{Y$ : $\left.Y \otimes_{B} M=0\right\}$.

A tilting module $M$ is said to be a separating (respectively, splitting) tilting module if the torsion theory $(\mathcal{T}(M), \mathcal{F}(M))$ splits in $\bmod A$ (respectively, the torsion theory $(\mathcal{X}(M), \mathcal{Y}(M))$ splits in $\bmod B)$.

For a general reference for tilting theory we refer the reader to $[A s],[R]$ and $[\mathrm{HR}]$.

We recall that the module $M$ is a *-module, as defined in [C], when the functor $\operatorname{Hom}_{A}(M,-)$ induces an equivalence of categories between $\operatorname{Gen} M$ and $\operatorname{Cogen} D_{B} M$.

\section{The transpose of A tilting module}

In this section we use results of the subcategories $C_{i}^{M}$ to prove that the transpose ${ }_{A}\left(\operatorname{Tr} M_{A}\right)$ of a tilting module $M_{A}$ is a ${ }^{*}$-module and give also a necessary and sufficient condition for ${ }_{A}\left(\operatorname{Tr} M_{A}\right)$ to be a tilting module.

Furthermore, we apply this result to obtain that the transpose of a splitting or a separating tilting module without nonzero projective summands is a tilting module. 
We start by stating a theorem of D'Este and Happel $[\mathrm{DH}]$ which motivated this section.

Theorem 2.1. ([DH]) Let $M_{A}$ be an A-module. Then: $M_{A}$ is a ${ }^{*}$-module if and only if $M_{\bar{A}}$ is a tilting $\bar{A}$-module, where $\bar{A}=A / \operatorname{ann} M_{A}$.

It is well known that if $M_{A}$ is a tilting module and $B=\operatorname{End}\left(M_{A}\right)$, then ${ }_{B} M$ is also a tilting module and $\psi: A \rightarrow \operatorname{End}\left({ }_{B} M\right)$ defined by $\psi(a)(t)=t . a, t \in{ }_{B} M$, $a \in A$ is an isomorphism [HR, 2]. Moreover, we need the following result.

Lemma 2.2. Let $M_{A}$ be a tilting $A$-module and $B=\operatorname{End}\left(M_{A}\right)$. Then $\psi$ induces an isomorphism $\operatorname{ann}\left(\operatorname{Tr} M_{A}\right) \simeq P\left({ }_{B} M,_{B} M\right)$, where $P\left({ }_{B} M,_{B} M\right)$ is the set of the endomorphisms of ${ }_{B} M$ which factor through a projective module.

Proof. If $M_{A}$ is a tilting module then $\psi: A \rightarrow \operatorname{End}\left({ }_{B} M\right)$ is an isomorphism. By $[\mathrm{PP}, 4.1]$ we know that $A\left(\operatorname{Tr} M_{A}\right) \simeq_{A}\left(\operatorname{Tr}_{B} M\right)$. Then $a \in \operatorname{ann}_{A}\left(\operatorname{Tr} M_{A}\right)$ if and only if $a \in \operatorname{ann}_{A}\left(\operatorname{Tr}_{B} M\right)$. On the other hand, $a . x=\psi(a) \cdot x=\operatorname{Tr} \psi(a)(x)=0$ for $x \in$ $\operatorname{Tr}_{B} M$. Hence $a \in \operatorname{ann}_{A}\left(\operatorname{Tr} M_{A}\right)$ if and only if $\psi(a) \in \mathrm{P}\left({ }_{B} M,_{B} M\right)$.

We prove next that the transpose of a tilting module is a ${ }^{*}$-module, and using D'Este and Happel's result stated in Theorem 2.1 we prove the following theorem.

Theorem 2.3. Let $M_{A}$ be a tilting $A$-module and $B=\operatorname{End}\left(M_{A}\right)$. Then:

a) ${ }_{A}\left(\operatorname{Tr} M_{A}\right)$ is a ${ }^{*}$-module.

b) $\underline{\operatorname{End}\left({ }_{B} M\right)}\left(\operatorname{Tr} M_{A}\right)$ is a tilting $\underline{\operatorname{End}}\left({ }_{B} M\right)$-module.

c) ${ }_{A}\left(\operatorname{Tr} M_{A}\right)$ is a tilting module if and only if $P\left({ }_{B} M,{ }_{B} M\right)=0$.

Proof. a) It is proven in [PP, 3.8] that a module $M_{A}$ is a ${ }^{*}$-module if and only if $C_{0}^{M_{A}}=C_{1}^{M_{A}}$ and the functor $\operatorname{Hom}_{A}(M,-)$ is exact on $C_{0}^{M_{A}}$.

On the other hand, using that $A \simeq \operatorname{End}\left({ }_{B} M\right)$ and ${ }_{B} M$ is a tilting module, we get that $C_{0}^{\operatorname{Tr} M_{A}}=C_{1}^{\operatorname{Tr} M_{A}}$ and the functor $\operatorname{Hom}_{A}\left(\operatorname{Tr} M_{A},-\right)$ is exact on $C_{0}^{\operatorname{Tr} M_{A}}$, from $[\mathrm{PP}, 4.11]$ and $[\mathrm{PP}, 4.7]$ respectively. It follows that ${ }_{A}\left(\operatorname{Tr} M_{A}\right)$ is a ${ }^{*}$-module.

b) By a) we know that ${ }_{A}\left(\operatorname{Tr} M_{A}\right)$ is a ${ }^{*}$-module. Then from Theorem 2.1 and Lemma 2.2 we obtain that $\bar{A}\left(\operatorname{Tr} M_{A}\right)$ is a tilting $\bar{A}$-module, where $\bar{A}=A / \operatorname{ann}\left(\operatorname{Tr} M_{A}\right) \simeq$ $\operatorname{End}\left({ }_{B} M\right) / P\left({ }_{B} M,{ }_{B} M\right)$.

c) Assume that $P\left({ }_{B} M,_{B} M\right) \neq 0$. By Lemma $2.2, \operatorname{ann}_{A}\left(\operatorname{Tr} M_{A}\right) \neq 0$. Therefore ${ }_{A}\left(\operatorname{Tr} M_{A}\right)$ is not faithful and consequently ${ }_{A}\left(\operatorname{Tr} M_{A}\right)$ is not a tilting module.

The converse follows directly from $b$ ).

Lemma 2.4. Let $M_{A}$ be a tilting module. Then the number of isomorphism classes of indecomposable projective summands of $M_{A}$ is equal to the number of isomorphism classes of indecomposable projective summands of ${ }_{B} M$.

Proof. This result follows from the Connecting Lemma [HR, 2]. In fact, if $I, P$ are respectively the injective envelope and the projective cover of the simple module $S$, then $\operatorname{TrD} \operatorname{Hom}_{A}\left(M_{A}, I\right) \simeq \operatorname{Ext}_{A}^{1}\left(M_{A}, P\right)$. Moreover, $P \in \operatorname{add} M_{A}$ if and only

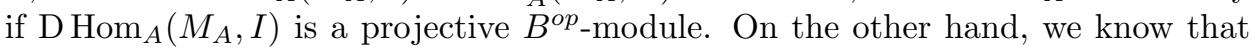
$D_{B} M=\operatorname{Hom}_{A}\left(M_{A}, D A\right)$, therefore $\mathrm{D} \operatorname{Hom}\left(M_{A}, I\right) \in \operatorname{add}_{B} M$. 
So to each indecomposable projective summand $P$ of $M_{A}$ we associate the indecomposable projective summand $\theta(P)=\mathrm{DHom}_{A}\left(M_{A}, I\right)$ of ${ }_{B} M$. Since $D A \in$ Gen $M_{A}$ and the functor $\left.\operatorname{Hom}_{A}\left(M_{A},-\right)\right|_{\text {Gen } M_{A}}$ is faithful, the correspondence $\theta$ is injective. By [HR, 2][As, 2.3] we know that each indecomposable projective module ${ }_{B} P$ is of the form $\mathrm{D} \mathrm{Hom}_{A}\left(M_{A}, I_{A}\right)$, with $I_{A}$ injective, so $\theta$ is also surjective.

Next, we give equivalent conditions for the transpose of a tilting module $M$ to be a tilting module. Let $\left(M_{A}\right)^{*}=\operatorname{Hom}_{A}\left(M_{A}, A\right)$.

Proposition 2.5. Let $M_{A}$ be a tilting module and $B=\operatorname{End}\left(M_{A}\right)$. Then the following conditions are equivalent:

a) ${ }_{A}\left(\operatorname{Tr} M_{A}\right)$ is a tilting module

b) $\left(M_{A}\right)^{*}=0$

c) $P\left(M_{A}, M_{A}\right)=0$

d) $\left(\operatorname{Tr}_{B} M\right)_{B}$ is a tilting module

e) $\left({ }_{B} M\right)^{*}=0$

f) $P\left({ }_{B} M,,_{B} M\right)=0$

Proof. To prove that a) implies b) we assume ${ }_{A}\left(\operatorname{Tr} M_{A}\right)$ is a tilting module. Then the module $M_{A}$ does not have projective summands and $\operatorname{id}\left(\operatorname{DTr} M_{A}\right) \leq 1$. That is to say, $\operatorname{Tr} \operatorname{Tr} M_{A} \simeq M_{A}$ and $\operatorname{Hom}_{A}\left(\operatorname{Tr} \operatorname{Tr} M_{A}, A\right)=0$. Then $\left(M_{A}\right)^{*}=0$.

Clearly b) implies c), and it follows directly from Theorem 2.3 that c) implies d). The remaining implications follow from the previous ones using that ${ }_{B} M$ is a tilting module.

As a consequence we obtain that the transpose of a separating (or splitting) tilting module with no projective summands is a tilting module. This result can also be obtained from the work of Hoshino [Ho,2].

Corollary 2.6. Let $M_{A}$ be a splitting (or separating) tilting module without nonzero projective summands and $B=\operatorname{End}\left(M_{A}\right)$. Then ${ }_{A}\left(\operatorname{Tr} M_{A}\right)$ is a tilting A-module.

Proof. By the above proposition we only need to prove that $\left({ }_{B} M\right)^{*}=0$. So we assume that this is not the case and consider a nonzero morphism $f::_{B} M \rightarrow B$. Then $D f: D B \rightarrow D_{B} M$ is also nonzero. Therefore there exist an indecomposable injective module $I_{B}$ and a nonzero morphism $h: I_{B} \rightarrow D_{B} M$, and since $D_{B} M \in$ $\mathcal{Y}(M)$ we conclude that $I_{B} \notin \mathcal{X}(M)$.

We assume first that the tilting module $M_{A}$ is splitting. Then $I_{B} \in \mathcal{Y}(M)$. By [As, 2.3] we conclude that there exists an injective module $I_{A}$ in $\bmod A$ such that $I_{B} \simeq \operatorname{Hom}_{A}\left(M, I_{A}\right)$, and from the Connecting Lemma we obtain $0=\operatorname{TrD} I_{B} \simeq$ $\operatorname{TrD} \operatorname{Hom}\left(M, I_{A}\right) \simeq \operatorname{Ext}_{A}^{1}\left(M, P_{0}\left(I_{A} / r I_{A}\right)\right)$. So the module $P_{0}$ belongs to $\mathcal{T}(M)$ and is a projective. Thus $P_{0} \in \operatorname{add} M_{A}$, contradicting our hypothesis.

The proof when $M_{A}$ is separating is similar.

\section{Modules $M_{A}$ Such that $C_{0}^{M_{A}}=C_{1}^{M_{A}}$ And tilting modules}

In this section we consider an $A$-module $M_{A}, B=\operatorname{End}\left(M_{A}\right)$ and we show that $M_{A}$ is a tilting module if and only if $D A \in C_{0}^{M_{A}}, C_{0}^{M_{A}}=C_{1}^{M_{A}}$ and $C_{0}^{B}{ }^{M}=C_{1}^{B}{ }^{M}$.

We start by studying the relation between the validity of some of the properties (T1), (T2) and (T3) defining a tilting module, and the conditions $C_{0}^{M_{A}}=C_{1}^{M_{A}}$ and $C_{0}^{B^{M}}=C_{1}^{B^{M}}$. 
It is well known that $C_{0}^{M_{A}}=\operatorname{Gen} M_{A}$ for any module $M_{A}$. By [PP, 3.12], when $M_{A}$ is a tilting module it satisfies the condition $C_{0}^{M_{A}}=C_{1}^{M_{A}}$. We will prove that the validity of (T2) and (T3) implies that $C_{0}^{M_{A}}=C_{1}^{M_{A}}$, and exhibit examples showing that no other combination of two of the properties (T1), (T2) and (T3) implies $C_{0}^{M_{A}}=C_{1}^{M_{A}}$.

We introduce now the following notation. For a module $M=M_{A}$, let $M^{\perp_{n}}=$ $\cap_{i=1}^{n} \operatorname{Ker}\left(\operatorname{Ext}_{A}^{i}(M,-)\right)$ and $M^{\perp}=\cap_{i>0} \operatorname{Ker}\left(\operatorname{Ext}_{A}^{i}(M,-)\right)$.

It is well known for a tilting module $M$ that $M^{\perp_{1}}=\operatorname{Gen} M$. In the next lemma we prove that it is enough to assume that $M$ satisfies (T3) for the inclusion $M^{\perp_{1}} \subseteq$ Gen $M$ to hold.

Lemma 3.1. If $M$ is an $A$-module and satisfies (T3) then $M^{\perp_{1}} \subseteq \operatorname{Gen} M$.

Proof. Since $M$ satisfies (T3) then there is an exact sequence $0 \rightarrow A \stackrel{f}{\rightarrow} M^{\prime} \rightarrow$ $M^{\prime \prime} \rightarrow 0$ with $M^{\prime}, M^{\prime \prime}$ in add $M$.

Assume now that $X \in M^{\perp_{1}}$. Let $f_{1}, \cdots, f_{n}$ be generators of the $Z(A)$-module $\operatorname{Hom}_{A}\left(M^{\prime}, X\right)$ and $\varphi=\left(f_{1}, \cdots, f_{n}\right)^{t}:\left(M^{\prime}\right)^{n} \rightarrow X$, where $(Z(A)$ denotes the center of $A$ ). We will prove that $\varphi$ is an epimorphism, so that $X \in$ Gen $M$.

By applying the functor $\operatorname{Hom}_{A}(-, X)$ to the above sequence we get the exact sequence:

$0 \rightarrow \operatorname{Hom}_{A}\left(M^{\prime \prime}, X\right) \rightarrow \operatorname{Hom}_{A}\left(M^{\prime}, X\right) \stackrel{\operatorname{Hom}(f, X)}{\longrightarrow} \operatorname{Hom}_{A}(A, X) \rightarrow \operatorname{Ext}_{A}^{1}\left(M^{\prime \prime}, X\right)=0$, and the commutative diagram

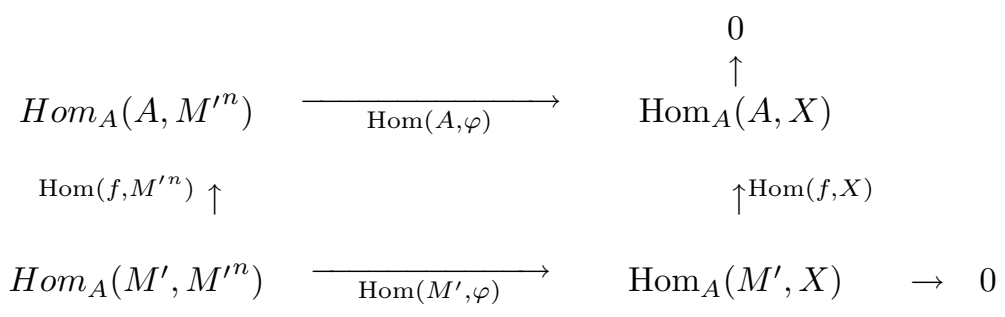

shows that $\operatorname{Hom}(A, \varphi)$ is an epimorphism. Terefore so is $\varphi$, and $X \in \operatorname{Gen} M$.

We will prove that if $M$ satisfies the conditions (T2) and (T3) then $C_{0}^{M}=C_{1}^{M}$. The converse is not true. In fact, any simple module $M$ satisfies $C_{0}^{M}=C_{1}^{M}$, and (T3) does not hold for $M$. Moreover, such a module $M$ can be chosen so that neither (T1) nor (T2) hold, as it is the case when $M$ is the simple module over the algebra $K[X] /\left(X^{2}\right)$.

Proposition 3.2. Let $M$ be an A-module. If $M$ satisfies (T2) and (T3) then $C_{0}^{M}=C_{1}^{M}$

Proof. Let $X$ in $C_{0}^{M}$ and consider an exact sequence $0 \rightarrow K \rightarrow M^{\prime} \rightarrow X \rightarrow 0$ with $M^{\prime} \in \operatorname{add} M$ such that the induced sequence $0 \rightarrow \operatorname{Hom}_{A}(M, K) \rightarrow \operatorname{Hom}_{A}\left(M, M^{\prime}\right) \rightarrow$ $\operatorname{Hom}_{A}(M, X) \rightarrow 0$ is exact. In order to prove that $C_{0}^{M}=C_{1}^{M}$ we only need to show that $K \in C_{0}^{M}$, by [PP, 3.5].

From the long exact sequence associated to $\operatorname{Hom}_{A}(M,-)$ and $0 \rightarrow K \rightarrow M^{\prime} \rightarrow$ $X \rightarrow 0$, and using the fact that $\operatorname{Ext}_{A}^{1}\left(M, M^{\prime}\right)=0$ because $M$ satisfies (T2), we get that $\operatorname{Ext}_{A}^{1}(M, K)=0$, so $K \in M^{\perp_{1}}$. On the other hand, we know from 
Lemma 3.1 that $M^{\perp_{1}} \subseteq$ Gen $M=C_{0}^{M}$, because $M$ satisfies (T3). We conclude that $K \in C_{0}^{M}$.

The next examples show that neither (T1) and (T2), nor (T1) and (T3) imply $C_{0}^{M}=C_{1}^{M}$.

Example 3.3. Let $A$ be the hereditary $K$-algebra given by the quiver:

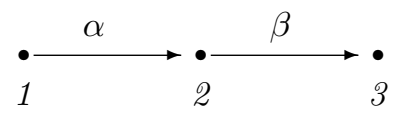

The Auslander-Reiten quiver $\Gamma_{A}$ is:

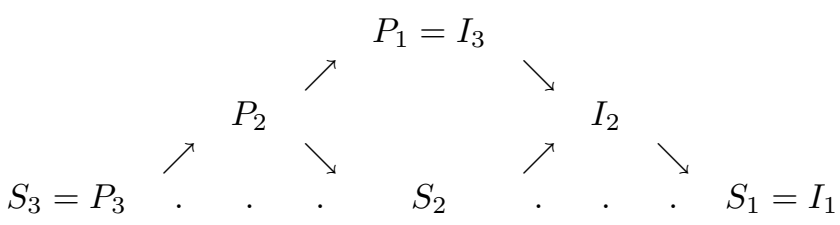

We consider the projective module $M=P_{2} \oplus P_{1}$. Clearly $M$ satisfies (T1) and (T2). We observe that $S_{2} \in C_{0}^{M}$ and $S_{2} \notin C_{1}^{M}$, so $C_{0}^{M} \neq C_{1}^{M}$.

Example 3.4. The module $M=P_{1} \oplus P_{2} \oplus I_{2}$ over the hereditary algebra of Example 3.3 has projective dimension 1 , satisfies (T3) and $C_{0}^{M} \neq C_{1}^{M}$.

Though, as we observed above, there are modules $M_{A}$ such that $C_{0}^{M}=C_{1}^{M}$ which do not satisfy any of the three properties of the definition of a tilting module, we will prove that the condition $C_{0}^{M}=C_{1}^{M}$ guarantees that the module ${ }_{B} M$ satisfies (T2), where $B=\operatorname{End}(M)$. To prove this result we need the following lemma.

Lemma 3.5. Let $M$ be an $A$-module such that $C_{0}^{M}=C_{1}^{M}$. Then $\operatorname{Im}\left(\operatorname{Hom}_{A}(M,-)\right)$ $\subseteq \operatorname{Ker}\left(\operatorname{Tor}_{1}^{B}\left(-,{ }_{B} M\right)\right)$.

Proof. It is proven in $[\mathrm{PP}, 3.7]$ that $\operatorname{Im}\left(\operatorname{Hom}_{A}(M,-)\right)=\operatorname{Ker}\left(\operatorname{Tor}_{1}^{B}\left(-,,_{B} M\right)\right)$ when $C_{0}^{M}=C_{1}^{M}$ under the additional hypothesis that the functor $\operatorname{Hom}_{A}(M,-)$ is exact in $C_{0}^{M}$. It is not difficult to see that without this additional hypothesis the argument used there applies to prove the desired inclusion.

Proposition 3.6. Let $M$ be an $A$-module and $B=\operatorname{End}(M)$. If $C_{0}^{M}=C_{1}^{M}$ then ${ }_{B} M$ satisfies (T2).

Proof. We consider the following natural isomorphisms:

$$
\operatorname{Ext}_{B}^{1}\left({ }_{B} M,_{B} M\right) \simeq \operatorname{DTor}_{1}^{B}\left(D_{B} M,_{B} M\right) \simeq \operatorname{DTor}_{1}^{B}\left(\operatorname{Hom}_{A}(M, D A),_{B} M\right) .
$$

By Lemma 3.5, $\operatorname{Tor}_{1}^{B}\left(\operatorname{Hom}_{A}(M, D A),_{B} M\right)=0$. Then $\operatorname{Ext}_{B}^{1}\left({ }_{B} M,_{B} M\right)=0$.

Let ${ }_{B} M_{A}$ be a $B$ - $A$-bimodule. We denote by $F=\operatorname{Hom}_{A}(M,-): \bmod A \rightarrow \bmod B$ and $G=-\otimes_{B} M: \bmod B \rightarrow \bmod A$ the pair of adjoint functors determined by $M$. Let $\epsilon_{X}$ denote the counit and $\mu_{Y}$ the unit of the adjunction, for $X \in \bmod A$ and $Y \in \bmod B$. We recall that $F\left(\epsilon_{X}\right) \mu_{F X}=i d_{F X}$ and $\epsilon_{G Y} G\left(\mu_{Y}\right)=i d_{G Y}$.

In $[\mathrm{PP}, 2.2]$ we proved that if $\epsilon_{M}: G F M \rightarrow M$ is an isomorphism then $C_{1}^{M} \subset$ $\operatorname{Im} G \subset C_{0}^{M}$. In the next lemma we show that if $B=\operatorname{End}(M)$ and $\mu_{D M}: D_{B} M \rightarrow$ $F G D_{B} M$ is an isomorphism then $D C_{1}^{{ }^{B}}{ }^{M} \subset \operatorname{Im} F \subset D C_{0}^{B^{M}}$. 
Lemma 3.7. Let $M$ be an $A$-module and $B=\operatorname{End}(M)$. Then:

i) $\operatorname{Im} F \subset D C_{0}^{B} M=\operatorname{Cogen} D_{B} M$

ii) If $\mu_{D_{B} M}: D_{B} M \rightarrow F G D_{B} M$ is an isomorphism then $D C_{1}^{B}{ }^{M} \subset \operatorname{Im} F$

iii) If $\mu_{D_{B} M}$ is an isomorphism, $C_{0}^{M}=C_{1}^{M}$ and $C_{0}^{B}{ }^{M}=C_{1}^{B}$ then $M$ is a ${ }^{*}$-module

Proof. i) Let $Y_{B}$ in $\operatorname{Im} F$ and $X^{\prime}$ be in $\bmod A$ such that $F X^{\prime}=Y_{B}$. Since any module $X$ can be immersed in an injective module, there is an exact sequence $0 \rightarrow X^{\prime} \rightarrow(D A)^{n}$, with $n \in N$. Applying the functor $F$ we get the exact sequence: $0 \rightarrow F X^{\prime} \rightarrow(F D A)^{n}$. Since $D_{B} M=F D A$ we obtain an exact sequence $0 \rightarrow$ $Y_{B} \rightarrow(D M)^{n}$, proving that $F X^{\prime}=Y_{B} \in \operatorname{Cogen} D_{B} M$.

ii) Let ${ }_{B} X \in C_{1}^{B}$, and let ${ }_{B} M_{1} \rightarrow_{B} M_{0} \rightarrow_{B} X \rightarrow 0$ be an exact sequence such that $\operatorname{Hom}_{B}\left({ }_{B} M,_{B} M_{1}\right) \rightarrow \operatorname{Hom}_{B}\left({ }_{B} M,_{B} M_{0}\right) \rightarrow \operatorname{Hom}_{B}\left({ }_{B} M,_{B} X\right) \rightarrow 0$ is exact.

By applying the duality we get exact sequences $0 \rightarrow D_{B} X \rightarrow D_{B} M_{0} \rightarrow D_{B} M_{1}$ and $0 \rightarrow \mathrm{D}^{H_{o m}}\left({ }_{B} M,_{B} X\right) \rightarrow \mathrm{D} \mathrm{Hom}_{B}\left({ }_{B} M,_{B} M_{0}\right) \rightarrow \mathrm{DHom}_{B}\left({ }_{B} M,_{B} M_{1}\right)$.

Since there is an isomorphism $\operatorname{D~Hom}_{B}\left({ }_{B} M,_{B} X\right) \simeq D_{B} X \otimes_{B} M=G D_{B} X$, natural in ${ }_{B} X$, we get that the sequence

$$
0 \rightarrow G D_{B} X \rightarrow G D_{B} M_{0} \rightarrow G D_{B} M_{1}
$$

is exact. We apply the functor $F$ and obtain a commutative diagram with exact rows

$$
\begin{array}{cccccc}
0 & \rightarrow & F G D_{B} X \\
\downarrow^{\mu_{D_{B}} X} & \rightarrow & F G D_{B} M_{0} & \rightarrow & F G D_{B} M_{1} \\
\downarrow^{\mu_{D_{B}} M_{0}} & & \downarrow^{\mu_{D_{B} M_{1}}} \\
0 & \rightarrow \quad D_{B} X & \rightarrow & D_{B} M_{0} & \rightarrow & D_{B} M_{1}
\end{array}
$$

where $\mu_{D_{B} M_{0}}$ and $\mu_{D_{B} M_{1}}$ are isomorphisms. So $\mu_{D_{B} X}$ is an isomorphism and therefore $D_{B} X \in \operatorname{Im} F$.

iii) Since $C_{0}^{M}=C_{1}^{M}$, the functor $F$ induces an equivalence of categories between Gen $M$ and $\operatorname{Im} F$, as follows from [PP, 3.1]. By hypothesis, i) and ii) we know that $D C_{1}^{B} M=\operatorname{Im} F=D C_{0}^{B} M=\operatorname{Cogen} D_{B} M$. Then $\operatorname{Im} F=\operatorname{Cogen} D_{B} M$, so $M$ is a *-module

Any tilting module $M$ satisfies that $C_{0}^{M}=C_{1}^{M}$ and it is well known that $D A \in$ Gen $M=C_{0}^{M}[\mathrm{HR}, 2]$. The converse is not true. We observe then that if $M$ is a tilting module and $B=\operatorname{End}(M)$, then ${ }_{B} M$ is also a tilting module, so that also $C_{0}^{B}{ }^{M}=C_{1}^{B}$. Now we prove the main result of this section.

Theorem 3.8. Let $M$ be an $A$-module and $B=\operatorname{End}(M)$. Then the following conditions are equivalent:

a) $M$ is a tilting module.

b) $C_{0}^{M}=C_{1}^{M}, C_{0}^{B}{ }^{M}=C_{1}^{{ }^{M}}$ and $D A \in C_{0}^{M}$.

Proof. We just observed that a) implies b). So we prove that b) implies a). We use the following characterization of tilting modules, given in [PP, 3.12]: the module $M$ is a tilting module if and only if $C_{0}^{M}=C_{1}^{M}$, the functor $F$ is exact in $C_{0}^{M}$ and 
$D A \in C_{0}^{M}$. We also recall from [PP, 3.8] that the first two properties characterize *-modules.

Thus to prove that b) implies a) we only need to show that a module $M$ satisfying b) is a ${ }^{*}$-module. This amounts to prove that $\mu_{D_{B} M}$ is an isomorphism, by Lemma 3.7, since we are assuming that $C_{0}^{M}=C_{1}^{M}$ and $C_{0}^{B}{ }^{M}=C_{1}^{B}{ }^{M}$. With this purpose we observe first that $\epsilon_{D A}$ is an isomorphism, because $D A \in C_{1}^{M}[\mathrm{PP}, 2.2]$. From the equality $1_{F D A}=F\left(\varepsilon_{D A}\right) \cdot \mu_{F D A}$ we get that $\mu_{F D A}$ is also an isomorphism. This proves that $\mu_{D_{B} M}$ is an isomorphism, since $F D A \simeq D_{B} M$, ending the proof of the theorem.

\section{Generalized tilting modules And the subcategory $C_{n}^{M}$}

In this section we study which of the results proven in section 3 for tilting modules can be extended to generalized tilting modules, as defined in $[\mathrm{M}]$ and $[\mathrm{H}, 3]$. In particular, we will prove that generalized tilting modules $M_{A}$ of projective dimension n satisfy that $C_{n-1}^{M_{A}}=C_{n}^{M_{A}}, C_{n-1}^{B}=C_{n}^{B}{ }^{M}$ and $\mathrm{D} A \in C_{n}^{M_{A}}$, for $B=$ $\operatorname{End}\left(M_{A}\right)$. However, the converse does not hold, so the characterization of tilting modules given in Theorem 3.8 can not be extended to generalized tilting modules.

We recall that a module $M \in \bmod A$ is a generalized tilting module if it satisfies the following conditions:

(TG1) $\operatorname{pd} M \leq n$

(TG2) $\operatorname{Ext}_{A}^{i}(M, M)=0$ for all $i \geq 1$

(TG3) There exists an exact sequence $0 \rightarrow A \rightarrow M_{0} \rightarrow M_{1} \rightarrow \cdots \rightarrow M_{n} \rightarrow 0$ with $M_{i} \in \operatorname{add} M$.

By [M, 1.16] and [H, 3] we know that if $M$ is a generalized tilting $A$-module and $B=\operatorname{End}\left(M_{A}\right)$ then ${ }_{B} M$ is a generalized tilting $B^{o p}$-module. We prove some relations between the validity of some of the properties defining a generalized tilting module, the subcategories $C_{i}^{M}$ and $M^{\perp_{i}}$. We denote $F=\operatorname{Hom}_{A}(M,-)$.

Proposition 4.1. Let $M$ be an $A$-module and $n, s \in \mathbb{N}$. Then:

a) If $M$ satisfies (TG2), $X \in C_{s-1}^{M}$ and $0 \rightarrow K_{s-1} \rightarrow M_{s-1} \rightarrow \ldots \rightarrow M_{0} \rightarrow$ $X \rightarrow 0$ is an exact sequence with $M_{i} \in$ addM and such that the induced sequence $0 \rightarrow F K_{s-1} \rightarrow F M_{s-1} \rightarrow \ldots \rightarrow F M_{0} \rightarrow F X \rightarrow 0$ is exact then $K_{s-1} \in M^{\perp_{s}}=$ $\cap_{i=1}^{s} \operatorname{KerExt}_{A}^{i}(M,-)$.

b) If $M$ satisfies (TG3) for $n$ then $M^{\perp_{n}} \subset C_{0}^{M}$.

c) If $M$ satisfies (TG2) and (TG3) for $n$ then $C_{n-1}^{M}=C_{n}^{M}$.

d) If $M$ satisfies (TG2) and (TG3) for $n$ then $M^{\perp} \subset \bigcap_{i \geq 0} C_{i}^{M}$.

e) If $M$ satisfies (TG1) and (TG2), then $C_{n-1}^{M} \subset M^{\perp}$, where $\operatorname{pd} M=n$

f) If $M$ is a generalized tilting A-module, and $\operatorname{pd} M=n$ then $M^{\perp}=C_{n-1}^{M}=C_{n}^{M}$.

Proof. We start by proving b). Assume $M$ satisfies (TG3) for $n$. Then there exists an exact sequence $0 \rightarrow A \rightarrow M_{0} \rightarrow M_{1} \rightarrow \cdots \rightarrow M_{n} \rightarrow 0$ with $M_{i} \in \operatorname{add} M$.

We denote $K_{j}=\operatorname{Ker}\left(M_{j} \rightarrow M_{j+1}\right)$ for $1 \leq j \leq n-1$. We get the exact sequences:

$$
\begin{gathered}
0 \rightarrow A \rightarrow M_{0} \rightarrow K_{1} \rightarrow 0, \\
0 \rightarrow K_{j} \rightarrow M_{j} \rightarrow K_{j+1} \rightarrow 0, \text { for } 1 \leq j \leq n-2 \\
0 \rightarrow K_{n-1} \rightarrow M_{n-1} \rightarrow M_{n} \rightarrow 0
\end{gathered}
$$


Let $X \in M^{\perp_{n}}$. By applying the functor $\operatorname{Hom}_{A}(-, X)$ to these sequences we obtain the exact sequence:

$0 \rightarrow \operatorname{Hom}_{A}\left(K_{1}, X\right) \rightarrow \operatorname{Hom}_{A}\left(M_{0}, X\right) \rightarrow \operatorname{Hom}_{A}(A, X) \rightarrow \operatorname{Ext}^{1}\left(K_{1}, X\right) \rightarrow 0$

and the isomorphisms $\operatorname{Ext}_{A}^{i}\left(K_{j}, X\right) \simeq \operatorname{Ext}_{A}^{i+1}\left(K_{j+1}, X\right)$ for $1 \leq j \leq n-2$ and $\operatorname{Ext}_{A}^{i}\left(K_{n-1}, X\right)=0$ for $1 \leq i \leq n-1$.

In particular $\operatorname{Ext}_{A}^{n-1}\left(\bar{K}_{n-1}, X\right)=0$. Then $\operatorname{Ext}_{A}^{1}\left(K_{1}, X\right) \simeq \operatorname{Ext}_{A}^{2}\left(K_{2}, X\right) \simeq$ $\cdots \simeq \operatorname{Ext}_{A}^{n-1}\left(K_{n-1}, X\right)=0$. Therefore $0 \rightarrow \operatorname{Hom}_{A}\left(K_{1}, X\right) \rightarrow \operatorname{Hom}_{A}\left(M_{0}, X\right) \rightarrow$ $\operatorname{Hom}_{A}(A, X) \rightarrow 0$ is exact. We deduce as in the proof of Lemma 3.1 that there exists an epimorphism $M^{\prime} \rightarrow X \rightarrow 0$. Then $X \in \operatorname{Gen} M=C_{0}^{M}$, proving b)

In order to prove the remaining items we consider $M$ satisfying (TG2), $X \in C_{s-1}^{M}$ and an exact sequence $0 \rightarrow K_{s-1} \rightarrow M_{s-1} \rightarrow \ldots \rightarrow M_{0} \rightarrow X \rightarrow 0$, whith $M_{i} \in$ add $M$ and such that the induced sequence $0 \rightarrow F K_{s-1} \rightarrow F M_{s-1} \rightarrow \ldots \rightarrow F M_{0} \rightarrow$ $F X \rightarrow 0$ is exact. Then

i) $\operatorname{Ext}_{A}^{j+s}\left(M, K_{s-1}\right) \simeq \operatorname{Ext}_{A}^{j}(M, X)$ for all $j \geq 1$ and

ii) $\operatorname{Ext}_{A}^{j}\left(M, K_{s-1}\right)=0$, for $1 \leq j \leq s$.

In fact, let $0 \rightarrow K_{0} \rightarrow M_{0} \rightarrow X \rightarrow 0$ be exact. Then $0 \rightarrow F K_{0} \rightarrow F M_{0} \rightarrow$ $F X \rightarrow 0$ is also exact, and $K_{0} \in C_{s-2}^{M}$. The long exact sequence associated to $0 \rightarrow$ $K_{0} \rightarrow M_{0} \rightarrow X \rightarrow 0$ yields $\operatorname{Ext}_{A}^{1}\left(M, K_{0}\right)=0$ and $\operatorname{Ext}_{A}^{j+1}\left(M, K_{0}\right) \simeq \operatorname{Ext}_{A}^{j}(M, X)$ for all $j \geq 1$, and i) and ii) follow then by induction on $\mathrm{s}$.

Now, the equalities in ii) mean precisely that that $K_{s-1} \in M^{\perp_{s}}$, proving a). If we also assume that (TG3) holds for $n$, then from a) and b) we get that $K_{n-1} \in$ $M^{\perp_{n}} \subseteq C_{0}^{M}$. Thus $M \in C_{n}^{M}$, proving c).

To prove d) we assume, moreover, that $X \in M^{\perp}$. Then from i) we obtain that $\operatorname{Ext}_{A}^{j+s}\left(M, K_{s-1}\right)=0$ for all $j \geq 1$. Using that $K_{s-1} \in M^{\perp_{s}}$ it follows that $K_{s-1} \in M^{\perp}$. On the other hand, since $M^{\perp} \subseteq C_{0}^{M}$, by b), we get that $K_{s-1} \in C_{0}^{M}$ and therefore $X \in C_{s}^{M}$, ending the proof of d).

e) Let $X \in C_{n-1}$, and assume that the above considered module $M$ satisfying (TG2) satisfies also (TG1) and has projective dimension $n$. Then $\operatorname{Ext}_{A}^{j+n}\left(M, K_{n-1}\right)=0$ for all $j \geq 1$ and from i) we get that $X$ is in $M^{\perp}$, as desired.

Finally, we observe that f) follows directly from c), d) and e).

Theorem 4.2. Let $M$ be a generalized tilting $A$-module, with $\operatorname{pd} M=n$ and $B=$ $\operatorname{End}\left(M_{A}\right)$. Then $C_{n-1}^{M_{A}}=C_{n}^{M_{A}}, C_{n-1}^{B}{ }^{M}=C_{n}^{B^{M}}$ and $D A \in C_{n}^{M_{A}}$

Proof. From Proposition $4.1 \mathrm{f}$ ) and the fact that both $M_{A}$ and ${ }_{B} M$ are generalized tilting modules we have that $C_{n-1}^{M_{A}}=C_{n}^{M_{A}}$ and $C_{n-1}^{B}=C_{n}^{B}$.

Since $D A$ is an injective module we know that $\operatorname{Ext}_{A}^{i}(M, D A)=0$, for all $i \geq 1$. Then $D A \in M_{A}^{\perp}$. By f), $C_{n}^{M_{A}}=M_{A}^{\perp}$, so $D A \in C_{n}^{M_{A}}$.

The converse of the theorem is not true as we illustrate in the following example.

Example 4.3. Let $A$ be the K-algebra given by the quiver

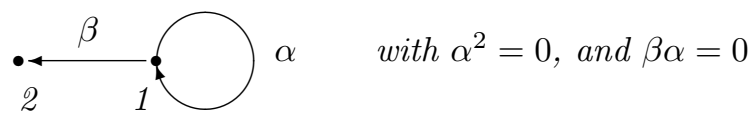


The Auslander-Reiten quiver $\Gamma_{A}$ is

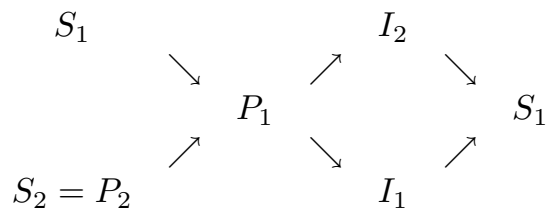

Let $M=I_{1} \oplus I_{2}$. Then $\operatorname{pd} M=\infty, D A \in C_{1}^{M}=C_{2}^{M}$ and $C_{1}^{B}{ }^{M}=C_{2}^{B} M$.

In the previous example we saw that the conditions $C_{n-1}^{M_{A}}=C_{n}^{M_{A}}, C_{n-1}^{B}{ }^{M}=C_{n}^{B} M$ and $D A \in C_{n}^{M_{A}}$ do not guarantee that $M$ is a generalized tilting module. We will prove that when $D A \in C_{n}^{M_{A}}$ and $C_{n-1}^{M_{A}}=C_{n}^{M_{A}}$ the module ${ }_{B} M$ satisfies (TG2).

We start with the following proposition.

Proposition 4.4. Let $M$ be an A-module and $n \geq 2$. If $X \in C_{n}^{M_{A}}$ then $\operatorname{Tor}_{i}^{B}(F X, M)=0$, for $i=1, \ldots, n-1$.

Proof. This result is proven in [PP, 3.7] under the additional hypothesis that $C_{0}^{M}=$ $C_{1}^{M}$, and the proof there can be easily addapted to the present situation, as we show next.

If $X \in C_{n}^{M_{A}}$ then there exists an exact sequence $M_{n} \rightarrow \cdots \rightarrow M_{0} \rightarrow X \rightarrow 0$ with $M_{i} \in \operatorname{add} M$, and such that the induced sequence $F M_{n} \rightarrow \cdots \rightarrow F M_{0} \rightarrow F X \rightarrow 0$ is exact. Denote $K_{j}=\operatorname{Ker}\left(M_{j} \rightarrow M_{j-1}\right)$ and $K_{0}=\operatorname{Ker}\left(M_{0} \rightarrow X\right)$. We get the exact sequences

(1) $0 \rightarrow F K_{0} \rightarrow F M_{0} \rightarrow F X \rightarrow 0$

(2) $0 \rightarrow F K_{j} \rightarrow F M_{j} \rightarrow F K_{j-1} \rightarrow 0$, for $1 \leq j \leq n$

We apply the functor $G=-\otimes_{B} M$ to the sequence (1) and consider the commutative diagram with exact rows

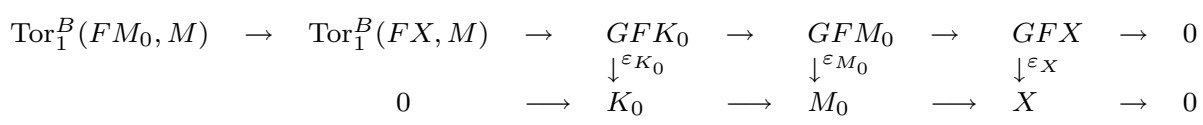

where $\varepsilon_{K_{0}}, \varepsilon_{M_{0}}$ and $\varepsilon_{X}$ are isomorphisms by [PP, 2.2] ( $K_{0} \in C_{1}^{M}$ because $\left.n \geq 2\right)$. Since $F M_{0}$ is $B$-projective, then $\operatorname{Tor}_{i}^{B}\left(F M_{0}, M\right)=0$. We obtain $\operatorname{Tor}_{1}^{B}(F X, M)=0$ and $\operatorname{Tor}_{i}^{B}\left(F K_{0}, M\right) \simeq \operatorname{Tor}_{i+1}^{B}(F X, M)=0$ for $i \geq 1$. The result follows then by induction, using that $K_{0} \in C_{n-1}^{M_{A}}$.

The previous result holds for all $i \geq 1$ under the additional hypothesis that $C_{n-1}^{M_{A}}=C_{n}^{M_{A}}, n \geq 1$, as we state next.

Corollary 4.5. Let $M$ be an A-module and $n \geq 1$. If $X \in C_{n}^{M_{A}}$ and $C_{n-1}^{M_{A}}=C_{n}^{M_{A}}$ then $\operatorname{Tor}_{i}^{B}\left(F X,_{B} M\right)=0$ for all $i \geq 1$.

Proof. If $C_{n-1}^{M_{A}}=C_{n}^{M_{A}}$, then $C_{n-1}^{M_{A}}=C_{m}^{M_{A}}$ for all $m \geq n \geq 1$. The result follows then from Proposition 4.4.

Corollary 4.6. Let $M$ be an $A$-module and $n \geq 1$. If $D A \in C_{n}^{M_{A}}$ and $C_{n-1}^{M_{A}}=C_{n}^{M_{A}}$ then $\operatorname{Ext}_{B}^{i}\left({ }_{B} M,{ }_{B} M\right)=0$ for all $i \geq 1$ ( ${ }_{B} M$ satisfies (TG2)). 
Proof. Since $D A \in C_{n}^{M_{A}}$ and $C_{n-1}^{M_{A}}=C_{n}^{M_{A}}$, we know by the above corollary that $\operatorname{Tor}_{i}^{B}(F D A, M)=0$ for all $i \geq 1$. Then $\operatorname{Tor}_{i}^{B}\left(D M,_{B} M\right)=0$ because $F D A=D M$. The corollary follows from the isomorphism $\operatorname{Ext}_{B}^{i}(M, M) \simeq \operatorname{Tor}_{i}^{B}(D M, M)$.

\section{ACKNOWLEDGEMENTS}

The authors gratefully acknowledge partial support from Universidad Nacional del Sur and from CONICET, of Argentina. The first author is a researcher from CONICET

\section{REFERENCES}

[As] I. Assem. Tilting theory. An introduction. Topics in Algebra, Banach Centre Publications, vol. 26, PWN, Warsaw, 127-180, (1990).

[A] M. Auslander Notes on representation theory. Brandeis Univ., (1973).

[ARS] M. Auslander, I. Reiten, and S. O. Smal $\emptyset$. Representation theory of artin algebras, vol. 36 of Cambridge Studies in Advanced Mathematics. Cambridge University Press, (1995).

[BB] S. Brenner and M. C. R. Butler. Generalization of the Bernstein-Gelfand-Ponomarev reflection functors. Lecture Notes in Math., 832, Springer-Verlag, Berlin-Heidelberg, New York, 103-169, (1980).

[C] R. Colpi. Some remarks on equivalences between categories of modules. Comm. Algebra 18(6), 1935-1951, (1990).

[DH] G. D'Este and D. Happel. Representable equivalences are represented by tilting modules. Rend. Sem. Mat. Univ. Padova, Vol. 83. (1990).

[H] D. Happel. Triangulated categories in the representation theory of finite dimensional algebras. London Math. Soc. Lecture Note Series. 119, (1988).

[HR] D. Happel and C.M. Ringel. Tilted algebras. Trans. Amer. Math. Soc., 284, 399-443, (1982).

[Ho] M. Hoshino. Splitting torsion theories induced by tilting modules. Comm. Algebra 11, $\mathrm{N}^{\circ} 4,427-441,(1983)$.

[M] Y. Miyashita Tilting modules of finite projective dimension. Math. Z., 193, 113-146, (1986).

[PP] M. I. Platzeck and N. I. Pratti. On a theorem of Auslander and applications. Comm. in Algebra, 28(6), 2817-2835, (2000)

[R] C. M. Ringel. Tame algebras and integral quadratic forms, Lecture Notes in Math., 1099, Springer-Verlag, Berlin-Heidelberg, (1984). 